\title{
CORRELAÇÃO ENTRE VOCABULÁRIO EXPRESSIVO E EXTENSÃO MÉDIA DO ENUNCIADO EM CRIANÇAS COM ALTERAÇÃO ESPECÍFICA DE LINGUAGEM
}

\section{Correlation between expressive vocabulary and Mean Length Utterance in children with Language Disorder}

\author{
Debora Maria Befi-Lopes ${ }^{(1)}$, Camila de Oliveira Nuñes ${ }^{(2)}$, Ana Manhani Cáceres ${ }^{(3)}$
}

\begin{abstract}
RESUMO
Objetivos: verificar a influência da idade no desempenho lexical e gramatical, e investigar a existência de correlação entre vocabulário expressivo e as medidas de extensão média de enunciado em crianças com alteração específica de linguagem. Método: participaram do estudo trinta sujeitos com diagnóstico de alteração específica de linguagem, entre 4:0 a 6:11 anos, sendo dez de cada faixa etária. Todos realizaram de forma completa a prova de vocabulário expressivo (ABFW) e de Extensão Média do Enunciado, independente de gênero ou escolaridade. O estudo foi retrospectivo e a coleta de dados se baseou nas filmagens e gravações das provas acima mencionadas. Resultados: o desempenho gramatical não apresentou diferença estatística entre as idades, mas foi observada correlação positiva entre o vocabulário expressivo e o uso de palavras de classe fechada, e entre o vocabulário expressivo e a extensão de palavras por enunciado ( $p$-valor $<0,05$ ). Conclusão: a idade isolada não é capaz de predizer o aprimoramento do vocabulário e da gramática, porém a expansão do vocabulário de substantivos favorece o aumento do número de palavras por sentença e o uso de palavras com função exclusivamente gramatical.
\end{abstract}

DESCRITORES: Linguagem Infantil; Transtornos de Linguagem; Testes de Linguagem; Vocabulário

\section{INTRODUÇÃO}

Alterações durante o desenvolvimento da linguagem podem manifestar-se em diferentes quadros do desenvolvimento infantil; porém, em alguns casos não caracterizam alterações globais e são denominadas alterações específicas de

(1) Fonoaudióloga; Livre-Docente do Curso de Fonoaudiologia da Faculdade de Medicina da Universidade de São Paulo - FMUSP; Coordenadora do Laboratório de Investigação Fonoaudiológica em Desenvolvimento da Linguagem e suas Alterações; Professora Associada do Departamento de Fisioterapia, Fonoaudiologia e Terapia Ocupacional da FMUSP. São Paulo, SP, Brasil.

(2) Fonoaudióloga graduada no Curso de Fonoaudiologia da Faculdade de Medicina da Universidade de São Paulo FMUSP; São Paulo, SP, Brasil.

(3) Fonoaudióloga; Assistente do Curso de Fonoaudiologia da Faculdade de Medicina da Universidade de São Paulo FMUSP; Doutoranda em Ciências da Reabilitação com ênfase em Comunicação Humana; São Paulo, SP, Brasil.

Conflito de interesses: inexistente linguagem (AEL). Estas abrangem os retardos de linguagem $(R L)$ e os distúrbios específicos de linguagem (DEL) ${ }^{1}$.

Nos casos de RL, observa-se que a aquisição das habilidades linguísticas ocorre na sequência típica, porém de forma lentificada. Já no DEL, a sequência do desenvolvimento apresenta desvios e o prejuízo no desempenho é acentuado ${ }^{1}$.

Um aspecto importante no diagnóstico diferencial entre os dois quadros é o fato de que no DEL a heterogeneidade das manifestações linguísticas é evidente, sendo difícil encontrar duas crianças com os mesmos prejuízos nas habilidades de linguagem ${ }^{2}$. Além disso, a literatura recomenda que a diferenciação entre os quadros seja realizada a partir da manutenção dos déficits linguísticos após os cinco anos ${ }^{3}$.

A avaliação destas crianças deve atentar aos comportamentos e habilidades linguísticas e comparar seu desempenho com valores de 
referência de crianças com desenvolvimento normal de mesma faixa etária².

Dentre os aspectos que devem ser observados nessa população, dois dos mais importantes são o vocabulário e a gramática. Na avaliação do vocabulário, que engloba as modalidades receptiva e expressiva, de maneira geral, as crianças com AEL apresentam desempenho melhor nas provas de vocabulário receptivo do que expressivo ${ }^{4}$.

Crianças brasileiras em desenvolvimento normal de linguagem, entre 2 e 4 anos de idade, utilizam mais verbos que substantivos em suas produções espontâneas desde o segundo ano de vida ${ }^{5}$. Além disso, com o aumento da idade, aprimoram o emprego da morfologia verbal, demonstrando evolução gradual no domínio da língua Portuguesa falada no Brasil ${ }^{6,7}$.

Apesar de não estar absolutamente esclarecida a relação entre o vocabulário e a gramática, o léxico parece preceder a gramática e o desenvolvimento de ambos parece ocorrer de forma sincrônica ao longo dos primeiros anos de vida ${ }^{8}$.

Em crianças em desenvolvimento típico é nítido que com a idade ocorra a expansão do vocabulário. Esta relação também foi encontrada nos quadros de AEL, porém de forma mais discreta ${ }^{9}$, além da melhora na recepção tornar-se gradualmente mais perceptível que na expressão ${ }^{4}$.

Estudos recentes apontam que a restrição do vocabulário nas AEL seja decorrente de falhas no processamento da informação linguística ${ }^{10}$, na memória operacional ${ }^{11}$ e na representação semântica ${ }^{12}$.

As dificuldades gramaticais são apontadas como uma das principais marcas clínicas nas AEL, uma vez que essas crianças demonstram desempenho inferior ao que seria esperado tanto no que se refere à idade cronológica quanto linguística ${ }^{13}$.

A literatura nacional apresenta a Extensão Média de Enunciado (EME) $)^{14}$, medida adaptada do Mean Length of Utterance (MLU) ${ }^{15}$, como estratégia para avaliar a estrutura gramatical da produção oral espontânea da criança e descrever as classes de palavras mais frequentes, os morfemas e as estruturas sintáticas utilizadas.

Em estudos que utilizaram esta medida verificou-se que as crianças com desenvolvimento típico tiveram melhor desempenho no vocabulário associado à melhora de desempenho gramatical, sendo que a ampliação lexical foi menor nas crianças com $A E L^{16}$. Assim, é possível sugerir que o déficit gramatical decorra de outros fatores, como déficits lexicais, dificuldade de processamento da informação linguística, falhas de compreensão e do aprendizado das regras sintáticas.
Sendo assim, estas crianças demonstram prejuízos que envolvem a extensão do enunciado, a aquisição e utilização das classes de palavras, e a morfologia ${ }^{17}$, o que parece significar que há uma limitação na habilidade de processamento gramatical ${ }^{18}$.

A partir do exposto, os objetivos do presente estudo foram (a) verificar a influência da idade no desempenho lexical e gramatical, e (b) investigar a existência de correlação entre vocabulário expressivo e as medidas de extensão média de enunciado em crianças com alteração específica de linguagem.

\section{MÉTODO}

Participaram dessa pesquisa 30 crianças pertencentes à faixa etária de 4 a 6:11 anos, sendo dez em cada idade. Todas foram diagnosticadas com alteração específica de linguagem segundo os critérios internacionais de inclusão no quadro, a saber: alteração de linguagem na ausência de deficiência auditiva, disfunção neuromotora, deficiência mental, déficit de atenção e hiperatividade, transtornos invasivos do desenvolvimento, distúrbios psicóticos, insultos cerebrais adquiridos, interação social restrita e distúrbio do comportamento e emocionais significativos ${ }^{1}$.

Os materiais utilizados foram os protocolos de avaliação das provas de vocabulário do $\mathrm{ABFW}^{19}$ e da avaliação da Extensão Média dos Enunciados $^{14}$, uma televisão, aparelho de DVD e as fitas com as gravações das avaliações para segunda transcrição.

A prova de vocabulário expressivo do $\mathrm{ABFW}^{19}$ permite a análise do desenvolvimento semântico e considera os substantivos divididos em nove campos semânticos. A análise considera as respostas no que se refere a designações verbais usuais (DVU), não designações (ND) e processos de substituição (PS).

A Extensão Média do Enunciado ${ }^{14}$ considera os morfemas gramaticais de palavras de classe aberta (MG-1), incluindo substantivos, verbos e artigos; os morfemas gramaticais de palavras de classe fechada (MG-2), incluindo preposições, pronomes e conjunções; a extensão média do enunciado em morfemas (EME-m), constituído pela média de morfemas por enunciado produzido; e extensão média de enunciado em palavras (EME-p), constituído pela média de palavras por enunciado ${ }^{17}$.

Por tratar-se de estudo retrospectivo, para a coleta dos dados foi realizada a consulta aos prontuários dos indivíduos atendidos no serviço de Fonoaudiologia da instituição em que o estudo foi desenvolvido. A seleção dos sujeitos foi baseada tanto na faixa etária, quanto no fato de terem 
realizado de forma completa as provas referidas, independente de gênero ou escolaridade. As filmagens e gravações de cada uma das avaliações dos sujeitos foram então acessadas e as provas foram transcritas novamente por uma das autoras.

Os dados obtidos foram tabulados para posterior análise, separando os valores: designações verbais usuais da prova de vocabulário expressivo (DVU), Extensão Média do Enunciado em morfemas (MG-1, MG-2 e EME-m) e Extensão Média do Enunciado em palavras (EME-p).

O presente estudo faz parte de um projeto maior já aprovado pela Comissão de Ética para Análise de Projetos de Pesquisa da Diretoria Clínica do Hospital das Clínicas da Faculdade de Medicina da Universidade de São Paulo sob número 1270/05.
Para comparar o desempenho das crianças com AEL foi utilizado o coeficiente de Correlação de Spearman, e o teste de Kruskal-Wallis foi utilizado para verificar se a idade influenciaria o desempenho. Ambos os testes são não-paramétricos, visto que o teste de Kolmogorov-Smirnov ( $p$-valor $<0,05)$ indicou que a distribuição dos dados não respeita a normalidade. O nível de significância adotado foi de $5 \%$ para todas as análises estatísticas.

\section{RESULTADOS}

A estatística descritiva dos dados é apresentada tanto para cada uma das faixas etárias, quanto para o grupo como um todo (Tabela 1).

Tabela 1 - Estatística descritiva do desempenho no vocabulário e na extensão média do enunciado

\begin{tabular}{|c|c|c|c|c|c|c|}
\hline & & $\mathbf{n}$ & Mínimo & Máximo & Média & $\begin{array}{l}\text { Desvio- } \\
\text { padrão }\end{array}$ \\
\hline \multirow{4}{*}{ DVU } & 4 anos & 10 & 35,5 & 69,5 & 56,0 & 10,24 \\
\hline & 5 anos & 10 & 36,4 & 72,0 & 54,9 & 10,48 \\
\hline & 6 anos & 10 & 48,3 & 75,4 & 63,5 & 8,63 \\
\hline & & 30 & 35,5 & 75,4 & 58,1 & 10,25 \\
\hline \multirow{4}{*}{ MG-1 } & 4 anos & 10 & 285 & 512 & 393,2 & 67,68 \\
\hline & 5 anos & 10 & 246 & 784 & 445,5 & 191,71 \\
\hline & 6 anos & 10 & 204 & 889 & 441,7 & 192,62 \\
\hline & & 30 & 204 & 889 & 426,8 & 157,89 \\
\hline \multirow{4}{*}{ MG-2 } & 4 anos & 10 & 64 & 142 & 100,9 & 21,32 \\
\hline & 5 anos & 10 & 45 & 237 & 101,6 & 68,50 \\
\hline & 6 anos & 10 & 39 & 321 & 122,4 & 88,05 \\
\hline & & 30 & 39 & 321 & 108,3 & 64,08 \\
\hline \multirow{4}{*}{ EME-m } & 4 anos & 10 & 3,81 & 6,54 & 4,9 & 0,82 \\
\hline & 5 anos & 10 & 2,91 & 10,21 & 5,5 & 2,56 \\
\hline & 6 anos & 10 & 2,43 & 12,10 & 5,6 & 2,78 \\
\hline & & 30 & 2,43 & 12,10 & 5,4 & 2,17 \\
\hline \multirow{4}{*}{ EME-p } & 4 anos & 10 & 2,64 & 5,12 & 3,8 & 0,67 \\
\hline & 5 anos & 10 & 2,27 & 8,18 & 4,2 & 2,10 \\
\hline & 6 anos & 10 & 2,04 & 10,36 & 4,6 & 2,39 \\
\hline & & 30 & 2,04 & 10,36 & 4,2 & 1,84 \\
\hline
\end{tabular}

Legenda: DVU - Designações Verbais Usuais; MG-1 - Morfemas Gramaticais 1; MG-2 - Morfemas gramaticais 2; MG-T - Total de Morfemas Gramaticais; EME-m - Extensão Média do Enunciado para Morfemas; EME-p - Extensão Média do Enunciado para Palavras, $\mathrm{n}$ - número de sujeitos. 
Para verificar se a idade influencia o desempenho nestes parâmetros foi realizado o teste de Kruskal-Wallis. Os resultados indicam que para 0 vocabulário expressivo há apenas uma tendência de expansão com aumento da idade $(H=5,142$, p-valor 0,076), mas para as medidas gramaticais não há diferença estatística (Tabela 2). Isto significa que para esta população a idade isolada não é capaz de predizer o aprimoramento do vocabulário e da gramática.

Tabela 2 - Resultado do Kruskal-Wallis para a comparação entre as faixas etárias

\begin{tabular}{|c|c|c|c|}
\hline & & $\mathbf{H}$ & Valor de $p$ \\
\hline & 4 anos & & \\
\hline \multirow[t]{2}{*}{ DVU } & 5 anos & 5,142 & 0,076 \\
\hline & 6 anos & & \\
\hline & 4 anos & & \\
\hline \multirow[t]{2}{*}{ MG-1 } & 5 anos & 0,093 & 0,955 \\
\hline & 6 anos & & \\
\hline \multirow{3}{*}{ MG-2 } & 4 anos & & \\
\hline & 5 anos & 1,396 & 0,497 \\
\hline & 6 anos & & \\
\hline \multirow{3}{*}{ EME-m } & 4 anos & & \\
\hline & 5 anos & 0,072 & 0,965 \\
\hline & 6 anos & & \\
\hline \multirow{3}{*}{ EME-p } & 4 anos & & \\
\hline & 5 anos & 0,720 & 0,698 \\
\hline & 6 anos & & \\
\hline
\end{tabular}

Legenda: DVU - Designações Verbais Usuais; MG-1 - Morfemas Gramaticais 1; MG-2 - Morfemas gramaticais 2; EME-m - Extensão Média do Enunciado para Morfemas; EME-p - Extensão Média do Enunciado para Palavras, $\mathrm{H}$ - valor do teste de Kruskal-Wallis.

Para verificar se as medidas de vocabulário e de gramática se correlacionam nestas provas, foi utilizado o coeficiente de correlação de Spearman. Os resultados indicam a existência de correlação positiva apenas entre o desempenho no vocabulário expressivo (DVU) e nos morfemas gramaticais de palavras de classe fechada (MG-2), e entre o desempenho no vocabulário expressivo (DVU) e na extensão média de enunciado em palavras (EME-P). Logo, é possível sugerir que com a expansão do vocabulário de substantivos ocorre um aumento do número de palavras por sentença enunciada e o uso de palavras com função exclusivamente gramatical (Tabela 3 ).

Tabela 3 - Resultados da Correlação de Spearman para os dados analisados

\begin{tabular}{ccc}
\hline & $\mathbf{r}_{\mathbf{s}}$ & p-valor \\
\hline DVU x MG-1 & 0,082 & 0,334 \\
DVU x MG-2 & 0,357 & $0,027^{*}$ \\
DVU x EME-M & 0,148 & 0,217 \\
\hline DVU x EME-P & 0,323 & $0,041^{*}$ \\
\hline
\end{tabular}

Legenda: DVU - Designações Verbais Usuais; MG-1 - Morfemas Gramaticais 1; MG-2 - Morfemas gramaticais 2; EME-m - Extensão Média do Enunciado para Morfemas; EME-p - Extensão Média do Enunciado para Palavras. Resultados significantes estão expres$\operatorname{sos} \operatorname{com}\left({ }^{*}\right)$. 


\section{DISCUSSÃO}

Neste estudo a ampliação do vocabulário expressivo de substantivos está relacionada à ampliação do uso de sentenças mais extensas e ao uso de palavras de classe fechada, o que confirma que para o desenvolvimento sintático é fundamental o processo de aquisição de palavras ${ }^{10}$.

A literatura internacional refere que no princípio do processo de aquisição de linguagem, observa-se que as crianças têm maior facilidade em adquirir palavras de classe aberta (substantivos, verbos e adjetivos) devido ao seu significado mais concreto ${ }^{20}$ e sua maior frequência de ocorrência na língua ${ }^{10}$. Isso implica que inicialmente as crianças precisam de muita exposição aos itens lexicais para que haja o aumento do vocabulário, porém, começam a adquirir palavras de classe fechada devido à necessidade sintática de elementos de ligação frasal ${ }^{17}$.

Logo, é a partir da experiência na língua e da maturidade linguística que as crianças passam a abstrair o conhecimento adquirido e deixam de precisar do apoio concreto do significado, sendo capazes de compreender e expressar palavras abstratas, que só tem função dentro da frase ${ }^{8}$. Com maior domínio das diversas classes gramaticais, a criança passa a utilizá-las em sua fala espontânea, o que acarreta aumento de palavras por enunciado expresso, e comprova que o avanço do desenvolvimento da linguagem resulta no aumento da extensão e da complexidade das frases ${ }^{14}$.

Nas crianças com AEL, estas características da aquisição lexical podem estar alteradas, pois geralmente se observa atraso no desenvolvimento lexical, dificuldades na aquisição dos conceitos abstratos e na combinação de palavras para formação de sentenças ${ }^{10}$. Todavia, apesar destas crianças terem um atraso na aquisição lexical, elas seguem a mesma linha de desenvolvimento, pois, independente da faixa etária é necessário primeiro dominar conceitos abstratos para somente depois poder desenvolver as habilidades linguísticas mais refinadas.

Neste estudo é possível notar que palavras de classe fechada foram mais utilizadas quando as crianças com AEL eram capazes de produzir maior número de substantivos. Este fato concorda com um estudo anterior que observou aumento significante do uso de palavras de classe fechada entre as faixas etárias na análise da extensão média de enunciado, sendo que destas, os pronomes são os mais utilizados ${ }^{21}$.

É interessante notar que para a população deste estudo o vocabulário expressivo apresentou uma tendência de crescimento com a idade, porém para as medidas gramaticais não houve indicativo de melhora no desempenho. Este fato já poderia ser esperado, uma vez que as crianças com AEL apresentam dificuldades morfossintáticas persistentes após os 4 anos de idade ${ }^{17}$. Tal persistência ocorre, pois conforme o avanço da idade, as crianças apresentam maior dificuldade nas habilidades linguísticas, visto que a exigência ambiental aumenta, demonstrando um desempenho mais instável e confirmando o quadro de DEL ${ }^{1}$.

Assim, nota-se que a idade não é suficiente para facilitar o desenvolvimento gramatical, mas será preciso a integração do conhecimento linguístico. Este achado concorda com um estudo que verificou que o aprendizado de morfemas, a partir de intervenção terapêutica, é facilitado pelo maior domínio da concordância verbal e nominal ${ }^{22}$. Todavia, sabe-se que as crianças com AEL encontram dificuldades em integrar os aspectos lexicais e gramaticais $^{23}$, o que torna tal desenvolvimento mais lento.

De maneira geral, a evolução das habilidades linguísticas nas crianças com AEL ocorre num padrão mais lentificado, sendo assim, o desenvolvimento não pode ser verificado a partir do aumento da idade, mas deve ser realizado com base na capacidade de lidar com demandas mais complexas e no próprio desenvolvimento linguístico.

Destaca-se ainda, que em decorrência das faixas etárias estudadas, é possível neste grupo existam casos de RL e outros de DEL. Por isso, para confirmação dos achados, seria interessante dar continuidade à pesquisa, incluindo crianças mais velhas, de maneira que todo o grupo esteja acima dos cinco anos.

\section{CONCLUSÃO}

A partir dos resultados é possível concluir que para a população estudada o desenvolvimento etário não prediz o desenvolvimento linguístico como no caso das crianças típicas, mas tal desenvolvimento decorre da expansão lexical, que favorece a ampliação da extensão frasal e o aprendizado das palavras de classe fechada, que possuem função exclusivamente gramatical e são adquiridas pela utilização da língua. 


\begin{abstract}
Purpose: to check the influence of age on the lexical and grammatical performance, and investigate the correlation between the number of usual verbal description in expressive vocabulary test and measures for mean length of utterance in children with Specific Language Impairment. Method: thirty children with Specific Language Impairment between 4:0 and 6:11 year-old took part in the study, and ten were selected from each age range that had the Expressive Vocabulary Test (ABFW) and Mean Length of Utterance Test, independent gender or education. The study was retrospective, based on filming and recordings of those tests as for the data collection. Results: no statistical difference was found in the grammatical performance among ages, but positive correlation was observed among the usual verbal description and parameters MG-2 and EME-P for Mean Length of Utterance Test $(p<0.05)$. Conclusion: age alone is not enough to predict improvement of vocabulary and grammar; however the expansion of nouns in vocabulary promotes the increase in the length of produced utterances and the use of grammar function words.
\end{abstract}

KEYWORDS: Child Language; Language Disorders; Language Tests; Vocabulary

\section{REFERÊNCIAS}

1. Reed V. Toddlers and preschoolers with specific language impairments. An introduction to children with language disorders. second ed. New York: Macmillan; 1994. p. 117-46.

2. Befi-Lopes D. Alterações no desenvolvimento da linguagem. Fonoaudiologia: Informação para a formação - Linguagem: Desenvolvimento normal, alterações e distúrbios. Rio de Janeiro: Guanabara Koogan; 2003. p. 19-32.

3. Bishop DV, Hayiou-Thomas ME. Heritability of specific language impairment depends on diagnostic criteria. Genes Brain Behav. 2008;7(3):365-72.

4. Befi-Lopes DM, Gândara JP, Felisbino FdS. Categorização semântica e aquisição lexical: desempenho de crianças com alteração do desenvolvimento da linguagem. Rev CEFAC. 2006;8(2):155-61.

5. Befi-Lopes DM, Cáceres AM, Araujo K. Aquisição de verbos em pré-escolares falantes do Português Brasileiro. Revista CEFAC. 2007;9(4):444-52.

6. Befi-Lopes DM, Cáceres AM. Verificação da morfologia verbal em pré-escolares falantes do Português Brasileiro. Revista da Sociedade Brasileira de Fonoaudiologia. 2009;14:470-5.

7. Befi-Lopes DM, Cáceres AM. Verb diversity analysis in the spontaneous speech of Brazilian preschoolers. Pro Fono. 2010;22(1):3-6.

8. Dixon JA, Marchman VA. Grammar and the lexicon: developmental ordering in language acquisition. Child Dev. 2007;78(1):190-212.

9. Hage SRdV, Pereira MB. Desempenho de crianças com desenvolvimento típico de linguagem em prova de vocabulário expressivo. Revista CEFAC. 2006;8(4):419-28.

10. Gândara JP, Befi-Lopes DM. Tendências da aquisição lexical em crianças em desenvolvimento normal e crianças com Alterações Específicas no Desenvolvimento da Linguagem. Rev Soc Bras Fonoaudiol. 2010;15(2):297-304.

11. Rodrigues A, Befi-Lopes DM. Phonological working memory and its relationship with language development in children. Pro Fono. 2009;21(1):63-8. 12. Befi-Lopes DM, Silva CP, Bento AC. Semantic representation and naming in children with specific language impairment. Pro Fono. 2010;22(2):113-8.

13. Owen AJ, Leonard LB. The production of finite and nonfinite complement clauses by children with specific language impairment and their typically developing peers. J Speech Lang Hear Res. 2006;49(3):548-71.

14. Araujo K, Befi-Lopes DM. Extensão Média do Enunciado de crianças entre 2 e 4 anos de idade: diferenças no uso de palavras e morfemas. Revista da Sociedade Brasileira de Fonoaudiologia. 2004;9(3):156-63.

15. Brown R. A first language the early stages Cambridge-Harvard University Press; 1973.

16. Rice ML, Redmond SM, Hoffman L. Mean length of utterance in children with specific language impairment and in younger control children shows concurrent validity and stable and parallel growth trajectories. J Speech Lang Hear Res. 2006;49(4):793-808.

17. Araujo K. Desempenho gramatical de criança em desenvolvimento normal e com Distúrbio Específico de Linguagem. São Paulo: Universidade de São Paulo; 2007. 
18. Sanz-Torrent M, Serrat E, Andreu L, Serra M. Verb morphology in Catalan and Spanish in children with specific language impairment: a developmental study. Clin Linguist Phon. 2008;22(6):459-74.

19. Befi-Lopes DM. Vocabulário. In: Andrade CRFd, Befi-Lopes DM, Fernandes FDM, Wertzner HF, editors. ABFW: teste de linguagem infantil nas areas de fonologia, vocabulário, fluência e pragmática. $2^{\circ}$ edição - revisada, ampliada e atualizada ed. Barueri: Pró-Fono; 2004. p. 33-50.

20. D'Odorico L, Fasolo M. Nouns and verbs in the vocabulary acquisition of Italian children. J Child Lang. 2007;34(4):891-907.
21. Araujo K. Aspectos do Desempenho Gramatical de Crianças Pré-Escolares em Desenvolvimento Normal de Linguagem. São Paulo: Universidade de São Paulo; 2003.

22. Pawłowska M, Leonard LB, Camarata SM, Brown $\mathrm{B}$, Camarata MN. Factors accounting for the ability of children with SLI to learn agreement morphemes in intervention. J Child Lang. 2008;35(1):25-53.

23. Leonard LB, Deevy P, Kurtz R, Krantz Chorev L, Owen A, Polite E, et al. Lexical aspect and the use of verb morphology by children with specific language impairment. J Speech Lang Hear Res. 2007;50(3):759-77.
http://dx.doi.org/10.1590/S1516-18462012005000017

RECEBIDO EM: 31/05/2011

ACEITO EM: 09/10/2011

Endereço para correspondência:

Debora Maria Befi-Lopes

Rua Cipotânea, 51 - Cidade Universitária

São Paulo - SP

CEP: 05360-160

E-mail: dmblopes@usp.br 